\title{
Correction to: Molecular targeted drugs resistance impairs double-strand break repair and sensitizes ER-positive breast cancer to PARP inhibitors
}

\author{
Yuna Suzuki $^{1} \cdot$ Wu Wenwen ${ }^{2} \cdot$ Tomohiko Ohta $^{2} \cdot$ Shin-ichi Hayashi ${ }^{1}$
}

Published online: 22 August 2021

(c) The Japanese Breast Cancer Society 2021

\section{Correction to: Breast Cancer \\ https://doi.org/10.1007/s12282-021-01282-5}

In the original publication of the article, the funding information should be revised as below.

Funding This study was supported by AstraZeneca K.K., Ministry of Education, Culture, Sports, Science and Technology of Japan, and Smoking Research Foundation.

Publisher's Note Springer Nature remains neutral with regard to jurisdictional claims in published maps and institutional affiliations.

The original article can be found online at https://doi.org/10.1007/ s12282-021-01282-5.

Shin-ichi Hayashi

shin@med.tohoku.ac.jp

1 Department of Molecular and Functional Dynamics, Graduate School of Medicine, Tohoku University, 2-1 Seoryo-machi, Aoba-ku, Sendai 980-8575, Japan

2 Department of Translational Oncology, St. Marianna University Graduate School of Medicine, Kawasaki, Japan 